\title{
Device-to-Device Communication Underlaying Cellular Communications Systems
}

\author{
Pekka JÄNIS ${ }^{1}$, Chia-Hao YU², Klaus DOPPLER ${ }^{3}$, Cássio RIBEIRO ${ }^{3}$, Carl WIJTING ${ }^{3}$, \\ Klaus HUGL ${ }^{3}$, Olav TIRKKONEN ${ }^{2}$, Visa KOIVUNEN ${ }^{1}$ \\ ${ }^{1}$ Department of Signal Processing and Acoustics, Helsinki University of Technology, Espoo, Finland \\ ${ }^{2}$ Department of Communications and Networking, Helsinki University of Technology, Espoo, Finland \\ ${ }^{3}$ Nokia Research Center, Nokia Group, Helsinki, Finland \\ Email: pekka.janis@tkk.fi
}

Received December 9, 2008; revised March 14, 2009; accepted March 18, 2009

\begin{abstract}
In this article we propose to facilitate local peer-to-peer communication by a Device-to-Device (D2D) radio that operates as an underlay network to an IMT-Advanced cellular network. It is expected that local services may utilize mobile peer-to-peer communication instead of central server based communication for rich multimedia services. The main challenge of the underlay radio in a multi-cell environment is to limit the interference to the cellular network while achieving a reasonable link budget for the D2D radio. We propose a novel power control mechanism for D2D connections that share cellular uplink resources. The mechanism limits the maximum D2D transmit power utilizing cellular power control information of the devices in D2D communication. Thereby it enables underlaying D2D communication even in interference-limited networks with full load and without degrading the performance of the cellular network. Secondly, we study a single cell scenario consisting of a device communicating with the base station and two devices that communicate with each other. The results demonstrate that the D2D radio, sharing the same resources as the cellular network, can provide higher capacity (sum rate) compared to pure cellular communication where all the data is transmitted through the base station.
\end{abstract}

Keywords: Peer-to-Peer, Device-to-Device, Power Control, Cellular Systems, IMT-Advanced

\section{Introduction}

Major effort has been spent in recent years on the development of next-generation wireless communication systems that will bring higher data rates and system capacity to end users and network operators. Examples of such next-generation systems are 3GPP Long Term Evolution (LTE) and WiMAX (see http://www.3gpp.org/ and http: //www.wimaxforum.org/).

Currently the evolution of such systems has been started under the scope of IMT-Advanced. In addition to traditional performance targets of high data rates and better coverage, the success of IMT-Advanced systems will depend on their ability to enable new services. It is expected that local services will contribute significantly to the growth of mobile communications. The widespread development of local services will be enabled by decreasing infrastructure costs and direct connectivity that supports peer-to-peer communication between local services and the end users. In fact already today mobile phones act as web server (see http://mymobilesite.net/) and offer direct connectivity, e.g. using Bluetooth technology.

In this article we propose to facilitate the local peer-topeer communication by a Device-to-Device (D2D) radio that operates as an underlay network to an IMT-Advanced cellular network. This D2D radio is a potential key enabler for low cost, seamless and high capacity local connectivity. We assume the infrastructure network to be a cellular network based on Orthogonal Frequency Division Multiple Access (OFDMA) technology. The cellular network operates in licensed bands, and it is important to guarantee that D2D transmissions will not generate harmful interference to cellular users. Similar 
problems are observed in the context of cognitive radios [1-3], where the cellular usage is the primary service.

In order to control the interference from D2D connections to the cellular network, we propose that the Base Station (BS) is able to control the maximum transmit power and the resources to be used for each D2D connection. Note that such a scenario is different from pure ad-hoc networks, without coordination from an infrastructure network, e.g. [4,5]. Further, we present a novel power control mechanism for D2D connections that share cellular uplink resources. The mechanism limits the maximum D2D transmit power, utilizing cellular power control information of the devices in D2D communication. The performance of D2D and cellular communications is evaluated by means of system simulations that include interference from multiple cells.

Secondly, we study a single cell scenario consisting of a device communicating with the BS and two devices that communicate with each other. We consider three modes of operation: D2D communication can share either uplink (UL) or downlink (DL) resources with the cellular network or use exclusive resources. If direct communication between the terminals is not beneficial, the two devices communicate through the BS of the cellular network. In semi-analytical studies we show that the D2D radio, sharing the same resources as the cellular network, can provide higher capacity (sum rate) than pure cellular communication through the BS.

This article is organized as follows: In Section 2 we present the motivation for mobile D2D communications with an example application and give a brief overview of the state of the art in D2D communication. In Section 3 we present the power control mechanism for the coexistence of D2D and cellular transmissions. In Section 4 we describe the simulation methodology and present the simulation results. In Section 5 the semi-analytical analysis is described and results are presented. In Section 6 we present results on indoor D2D connections sharing the DL resources with a metropolitan area network. In Section 7 we summarize our results and the conclusions are given.

\section{Mobile Device-to-Device Communication}

Next generation mobile communication systems such as 3 GPP LTE and WiMAX are optimized for wide area and metropolitan area operation. In recent years local area networks based on WLAN have been increasingly popular, as they enable access to the internet and to local services with low cost APs and cheap and fast access to wireless spectrum in the license exempt bands. However only a licensed band can guarantee a controlled interference environment and local service providers might prefer to pay a small amount of money to get access to licensed spectrum when the license exempt bands get crowded. Cellular operators may offer such cheap access to spectrum with controlled interference enabled by D2D communication as underlay to the cellular network.

This concept is illustrated in Figure 1, where UE denotes User Equipment. The BS allows UE2 and UE3 to communicate directly to each other while keeping some control over the D2D link to limit the interference to the cellular receiver. As an example, consider the case where a media server is put up at a rock concert from which visitors can download promotional material using the D2D connection. At the same time, the cellular network can handle phone calls and internet data traffic without the additional load that would be caused by traffic from the media server. The D2D operation itself can be transparent to the user. She simply enters a URL, the network would detect traffic to the media server and hand it over to a D2D connection. The same application could also be enabled by a media server with built in WLAN AP or Bluetooth. However in that case the user has to define the WLAN AP or perform Bluetooth pairing which can be tedious especially if a secure connection is required.

Compared to other local connectivity solutions based on for example Bluetooth or WLAN the D2D communication supported by a cellular network offers additional compelling advantages. First the network can advertise local services available within the current cell. Thus for automated service discovery, the devices do not have to constantly scan for available WLAN AP or Bluetooth devices. This is especially advantageous when considering that the constant scanning of Bluetooth devices or WLAN APs is often switched off to reduce the power consumption. Secondly, the cellular network can distribute encryption keys to both D2D devices so that a secure connection can be established without manual pairing of devices or entering encryption keys.

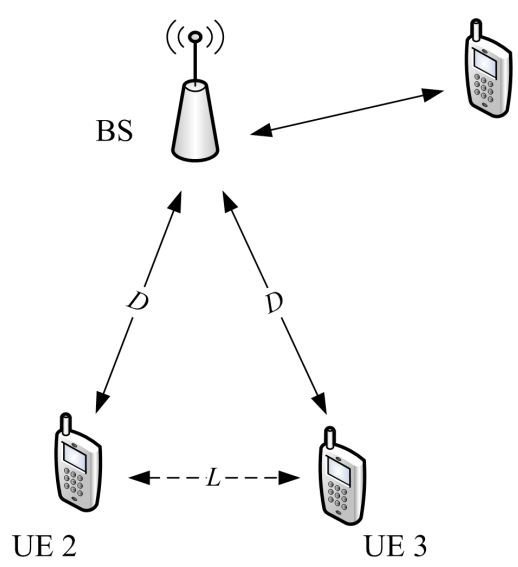

UE 1

Figure 1. Illustration of D2D communication as an underlay network to an infrastructure network. UE1 is a cellular user whereas UE2 and UE3 are D2D users. $D$ denotes the distance between D2D nodes and BS, and $L$ denotes the D2D link distance. 
Several wireless standards have addressed the need for D2D operation in the same band as the BS, also called Access Point (AP) or central controller. Examples of such standards are Hiperlan 2 [6], TETRA [7], and WLAN.

In all these standards D2D communication is assumed to occur on separate resources. For example, in standards employing Orthogonal Frequency Division Multiplexing (OFDM) as the physical layer, like Hiperlan 2, User Equipments (UE) involved in D2D communications are not allowed to share the same OFDM symbol with UEs communicating to the infrastructure network. This restriction limits the interference. However it leads to inefficient utilization of resources, especially for large system bandwidths. The resource utilization is even more inefficient in TETRA, where several frequency channels are country-wide reserved solely for D2D communication; reducing the resources available for cellular communication when no D2D communication is present.

In WLAN the UE senses the medium and transmits if the resources are free. A drawback of such a scheme is that the AP does not have a direct possibility of controlling the D2D links and providing assistance, which could prove highly beneficial for the network [8].

\section{D2D Power Control when Sharing UL Resources}

Since the D2D communication takes place as an underlay communication to the cellular OFDMA network, the interference from D2D communication to the cellular network has to be coordinated and the BS should be aware of ongoing D2D connections. The UEs in D2D connections are still associated to the $\mathrm{BS}$ and can receive for example cellular calls. Thus, we propose that the D2D link initialization and the allocation of OFDMA Resource Block (RB) to the D2D links is managed by the BS. Therefore, there is an immediate opportunity for the BS to reduce the interference between the cellular and D2D links. Such a scheme for sharing UL resources is proposed in this section. The power level of D2D transmitters is chosen based on the cellular UL power control information to limit the interference to the cellular BS.

The easiest way to restrict the D2D interference would be to mandate a predefined maximum power level to the D2D transmitters, and this level could be chosen such that the expected degradation in the cellular links stays at a tolerable level. However, such an approach would have to be designed for the worst case scenario and would lead to inefficient use of resources. As the D2D transmitter may be arbitrarily close to the cellular receiver, the power level thus determined is likely to be inadequate for establishment of reliable D2D links, other than for extremely short range communication. On the other hand, the power level of the D2D transmitter could be substantially higher if the network would have some means to determine how close the D2D transmitter is to the cellular receiver.

In fact the cellular BS has just the required information for controlling the interference from D2D transmitters to the cellular BS in case the D2D links share UL resources with the cellular network. The UL power control in a cellular network aims at reducing the dynamic range of signals received from multiple devices, i.e. to reduce the near-far effect. The BS may use the cellular UL power control framework in setting the D2D transmit power. To be more specific, let us consider the SINR of the UL cellular transmission in an isolated cell with ideal transceivers and flat fading channel. In this case the expression for the cellular UL SINR may be written as

$$
\xi=\frac{P_{1} C_{1}}{P_{2} c_{2}+\sigma_{w}^{2}}
$$

where $P_{1}$ and $P_{2}$ denote the transmit powers of the cellular and D2D UEs, $c_{1}$ and $c_{2}$ the corresponding link gains to the base station, and $\sigma_{w}^{2}$ the additive white Gaussian noise power. The base station has full control over the powers $P_{1}$ and $P_{2}$ in Equation (1), given the limitations on the transmit power range of the terminal and on the dynamic range of the power control specified for the radio interface.

Equation (1) implies that in case of ideal UL power control without the presence of a D2D transmitter $\left(P_{2}=0\right)$ and a target SNR of $P / \sigma_{w}^{2}$, the cellular power control target is $P_{1} C_{1}=P$.

In order to keep the interference to UL transmissions under control, the BS can signal the D2D transmitter to apply a power level such that $P_{2} C_{2}=P / B$, where $B$ is a backoff parameter. For large values of the backoff parameter $B, \mathrm{D} 2 \mathrm{D}$ transmissions cause very low interference to UL transmissions. However, a large $B$ implies a reduced range for the D2D link itself. We can avoid this limitation on the range of the D2D link by incorporating a power boosting factor $\alpha$ to the transmit power of the UL transmitter that compensates for the remaining interference from D2D transmissions. In this case, Equation (1) is modified to

$$
\xi^{\prime}=\frac{\alpha P_{1} c_{1}}{P_{2} c_{2}+\sigma_{w}^{2}}
$$

The power boosting value $\alpha$ is defined such that the received SINR from UL transmission in Equation (2) is equal to the target SNR, i.e. $\xi^{\prime}=P / \sigma_{w}^{2}$. Hence, substituting $P_{1} c_{1}=P$ and $P_{2} c_{2}=P / B$ into Equation (2), we obtain

$$
\alpha=\frac{P}{B \sigma_{w}^{2}}+1
$$

Naturally, in case of no UL transmissions, the D2D transmitter does not need to apply a power backoff, i.e. 
$B=1$. Conversely, no power boosting is needed in case of no D2D transmissions. In fact, in cases when only a subset of the RBs is used by D2D traffic, the power boosting is only applied to those UL transmissions that share the RBs with a D2D pair. As a result, the received $\mathrm{UL}$ power is non-uniform over the system bandwidth, which tends to increase the inter-RB interference caused by power amplifier nonlinearities and limited receiver dynamic range. Moreover, UL transmissions are not perfectly orthogonal due to the effects of non-ideal synchronization and wireless propagation environment. Therefore we limit the boosting values to $10 \mathrm{~dB}$, which we assume to be still manageable.

\section{Numerical Results on Coexistence of D2D Communication and Cellular Network}

In this section we study the coexistence of D2D communication links with an interference-limited cellular local area network. The D2D pair is sharing either UL or DL resources with the cellular links. The aim is to find out the achievable D2D link quality when giving priority to the cellular links. The study is carried out by static system simulations and empirical SINR distributions for both the cellular and D2D links are evaluated.

\subsection{Scenario and Channel Model}

The scenario and network layout resembles a local area indoor scenario, illustrated in Figure 2. Nine BS serve a whole floor of $100 \mathrm{~m}$ times $100 \mathrm{~m}$. The scenario incorporates small room, corridor like longer rooms and a large open area in the center. Similar elements can be typically found in shopping malls or office areas.

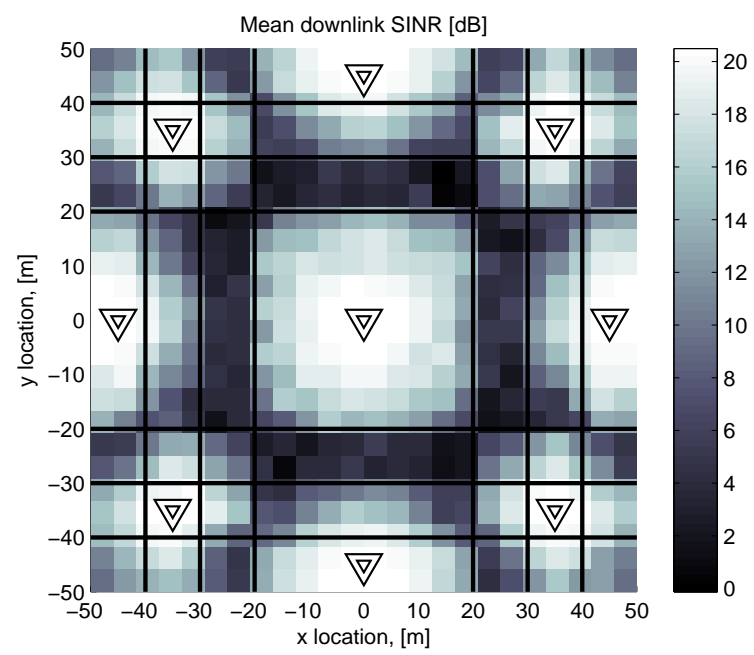

Figure 2. The simulated scenario for coexistence studies. The triangles represent the locations of base stations and the black horizontal and vertical lines represent walls. The color indicates the mean DL SINR without D2D interference as a function of location.
We have used the channel and propagation models defined in WINNER [9] scenario A1 (indoor/office) for our studies. Links in the same room have a distance dependent probability for Line-Of-Sight (LOS) conditions.

In the channel model, for the LOS and Non-LineOf-Sight (NLOS) propagation conditions, the path-loss exponent is 1.87 and 3.68, respectively, and the shadow fading standard deviation is $3 \mathrm{~dB}$ and $4 \mathrm{~dB}$, respectively. In addition, each wall introduces an additional attenuation of $5 \mathrm{~dB}$. Frequency selective fading is also modeled, with a resolution of $2 \mathrm{MHz}$.

The cellular UEs are uniformly distributed over the area and the locations of the D2D pairs are independent from the cellular UEs. The D2D pairs are generated with the restriction that the D2D link must reside within a single room.

\subsection{System Model}

We assume that the network operates on a $100 \mathrm{MHz}$ band using Time Division Duplexing (TDD). The base stations have acquired frame-synchronism and use the same split between UL and DL resources, such that there is no interference from neighboring cell DL transmission to UL transmissions or vice versa. The modulation scheme allows Frequency Division Multiplexing (FDM) transmissions from the BS to several UEs simultaneously, as well as Frequency Division Multiple Access (FDMA) for several UEs to the BS. Specifically, the $100 \mathrm{MHz}$ band is split into five orthogonal RBs of $20 \mathrm{MHz}$.

\subsubsection{Scheduler for Cellular Transmissions}

Each BS randomly selects five UEs to be scheduled, from those UEs that are not in D2D mode. For each UE it tries to allocate the RB with best SNR or the RB with second best SNR. If these are not available, the first free $\mathrm{RB}$ is allocated. In case there are less than five UEs associated to the $\mathrm{BS}$, the remaining free $\mathrm{RBs}$ are allocated to a UE with allocation in the adjacent RB. Hence, the network is fully loaded at every time instant. Since the channel is assumed reciprocal, the same frequency resources that are used for UL are also used for DL.

\subsubsection{UL Power Control}

For each BS, the total transmitted power is $25 \mathrm{dBm}$, which is evenly distributed over all sub-bands, i.e. $18 \mathrm{dBm}$ for each $20 \mathrm{MHz}$ band. For the UE, the uplink power control aims a target SNR of $P / \sigma_{w}^{2}$, limited by the maximum transmit power of $18 \mathrm{dBm}$ for the UE. With these settings, in the studied scenario about $10 \%$ of the UEs utilize maximum output power. When the UL power boosting defined in Section 3 is used, the portion of UEs reaching maximum transmit power increases to $40 \%$. 


\subsubsection{SINR Calculations}

The SINR of each $2 \mathrm{MHz}$ sub-band for a transmission originating from node $n$ and received by node $m$ is calculated as

$$
\xi_{n, m}=\frac{P_{n} c_{n, m}}{\sigma_{w}^{2}+\sigma_{\mathrm{Tx}}^{2}+\sigma_{\mathrm{Rx}}^{2}+I_{\mathrm{NL}}+\sum_{k \neq n} P_{k} c_{k, n}}
$$

where $I_{\mathrm{NL}}$ is the power of all out-of-band emissions at the receiver, and $\sigma_{w}^{2}, \sigma_{\mathrm{Tx}}^{2}$, and $\sigma_{\mathrm{Rx}}^{2}$ are the thermal noise power, the transmitter in-band distortion, and the receiver Analog-to-Digital Converter (ADC) noise floor, respectively, integrated over the $2 \mathrm{MHz}$ sub-bands. The thermal noise power is derived from the noise figure defined in LTE specifications for UE and BS [10]. With the chosen transmit power levels our network scenario is clearly interference-limited.

\subsection{Network Simulation Description}

The simulation arrangement is as follows. A large number of random independent snapshots of network operation, called drops, are modeled. For each drop, a set of cellular and D2D UEs are independently placed in the scenario. The path-loss and shadow fading values of each link are then determined and each cellular and D2D UE is associated with the BS to which it has the strongest link. Each cell has 5 cellular UEs and 5 D2D pairs, and the D2D transmissions are multiplexed to 5 sub-bands. This way we can ensure that each cellular transmission is interfered by exactly one intracell D2D link and vice versa. After all transmissions are scheduled, the transmit powers of the D2D transmitters are determined as in Section 3 and the SINR is computed as in Subsection 4.2.3.

\subsection{Results and Discussion}

In this section we present the simulation results on the SINR distributions for the cellular UL, cellular DL, and the D2D links. A wide range of transmit power levels without power control for the D2D link was simulated along with an UL power control based D2D case. The settings for the power control case have been chosen such that for $95 \%$ of the cellular links the SINR degradation is less than $3 \mathrm{~dB}$. This was achieved with a power backoff of $B=5 \mathrm{~dB}$, for a UL power control target SNR of $13 \mathrm{~dB}$. From Equation (3), this implies an UL power boost $\alpha=8.64 \mathrm{~dB}$.

From Figure 3(a) and 3(b), we observe that the maximum allowed D2D transmit power should be limited to $-10 \mathrm{dBm}$ in DL and $-24 \mathrm{dBm}$ in the UL phase of the frame, assuming that a $3 \mathrm{~dB}$ degradation of the cellular SINR at the 5-th percentile of the SINR CDF would be still tolerated. Assuming as well that a D2D link with $\operatorname{SINR} \geq 0$
$\mathrm{dB}$ is usable, we observe from 3(c) and 3(d) that the fraction of usable D2D links is $\approx 45 \%$ in DL phase and $\approx 33 \%$ in UL phase.

However, if the UL-based D2D power control scheme is applied, the percentage of usable links rises to $73 \%$ in UL phase while still maintaining the same cellular performance as for $-24 \mathrm{dBm}$ D2D transmit power, as observed in Figure 3(b) and 3(d).

As it can be appreciated from Figure 3 and from the discussion above, when the D2D transmit power is set to a fixed level such that the degradation to the cellular performance remains tolerable, D2D performance is slightly better when it uses DL resources than when it uses UL resources. This may sound counter-intuitive since, due to the fact that the BS's constant transmit power is significantly higher than the mean UL transmit power, the interference to the D2D links is higher in the DL than in UL phase. On the other hand, due to the same reason the cellular DL transmissions can tolerate much higher D2D transmit powers than the cellular UL transmissions. In the UL phase, the D2D transmit power must be set such that even in the event of a D2D transmitter being close to the BS the cellular performance does not degrade too much. Since UL power control guarantees that all UEs experience similar SINR in their UL transmissions regardless of their position in the network, this becomes a very strict requirement.

The proposed UL-based power control scheme effectively removes this restriction, resulting in significant improvement on the D2D performance. A similar power control scheme is not applicable in DL since the BS might schedule resources shared with D2D links to multiple cellular UEs. Each of these candidate cellular UEs would have to set a power control target to the D2D transmitters, implying significant overhead.

\section{Resource Allocation Analysis}

In the preceding sections we considered the coexistence of a D2D underlay and cellular network. Specifically, we demonstrated that it is possible to allow D2D communication to share the cellular resources and at the same time guarantee that the performance of the cellular communication links is not sacrificed, thus taking an approach where the cellular communication has priority. In this section we take a different viewpoint where neither the cellular nor the D2D communication have priority over the other. This gives insight on the maximum benefits in terms of overall performance that D2D underlay communication can provide.

We assume the Channel State Information (CSI) of all the involved links is available at BS so that the resource allocation decision of D2D users can be controlled centrally by the BS. The scenario where at most one cellular 


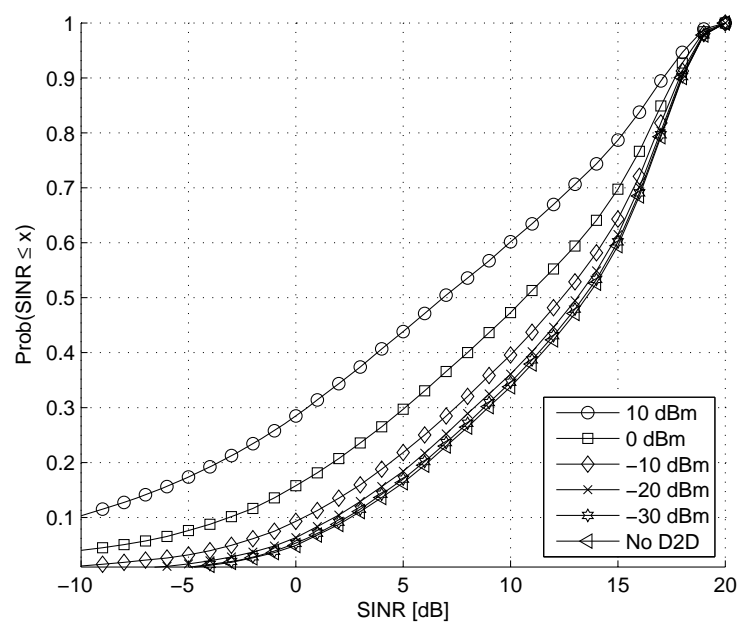

(a) DL SINR.

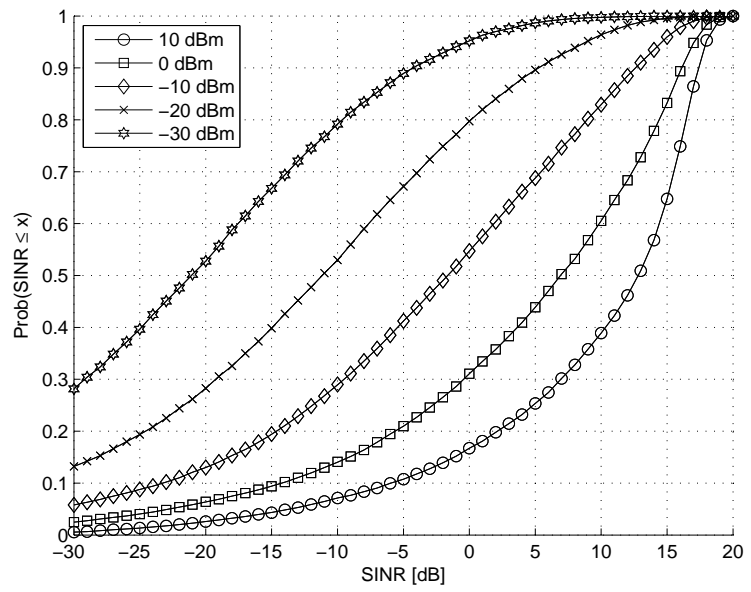

(c) D2D SINR in DL phase.

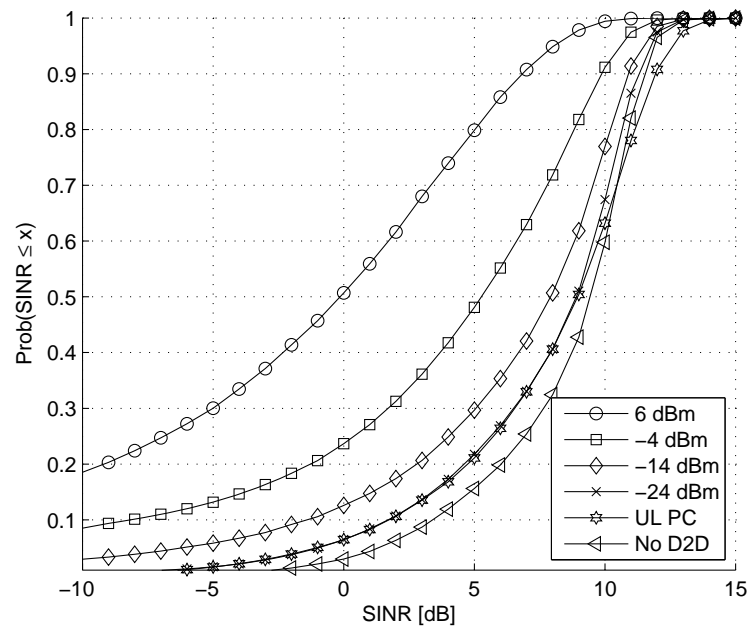

(b) UL SINR.

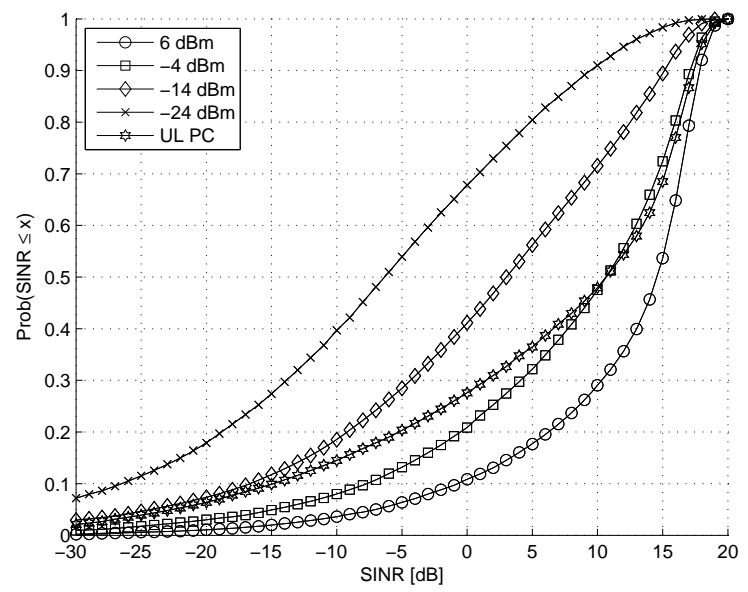

(d) D2D SINR in UL phase.

Figure 3. Empirical SINR CDFs in the local area scenario with various D2D transmitter power levels. The UL and DL SINR without D2D is shown for reference. The D2D SINR distributions are shown conditioned on the link distance being less than 8 meters. Each D2D link is within a single room.

user and one D2D communication pair will share the same radio resource is considered. Despite its simplicity, this scenario captures the minimum requirement for cellular communication and D2D communication to share the same resource.

In general, D2D communication causes no interference to the cellular users if they occupy separate resources. However, resource usage efficiency can be higher if the same resource is shared at the same time. We may achieve higher overall system performance if D2D and cellular communications co-exist in the same radio resource. We will discuss four different resource allocation modes including both separate and non-separate sharing schemes. They are illustrated in Figure 4 and detailed below:

- DL resource sharing (DLre): D2D communication happens in DL resources so that all the DL resources of the cellular user are interfered.
- UL resource sharing (ULre): Similar to DLre, D2D communication happens in UL resources, and all the UL resources of the cellular user are interfered.

- Separate resource sharing (SEPre): D2D communication takes half of the available resources from the cellular user, either from DL or UL resource. There is no interference between cellular and D2D communication.

- Cellular mode sharing (CellMod): The D2D users communicate with each other through the BS that acts like a relay node. They take half of the available resources either from the DL or the UL resources of the cellular user. Note that this mode is conceptually the same as traditional cellular system and is used as a reference.

In the following, we consider a normalized isolated circular cell (with radius equal to 1) as illustrated in 


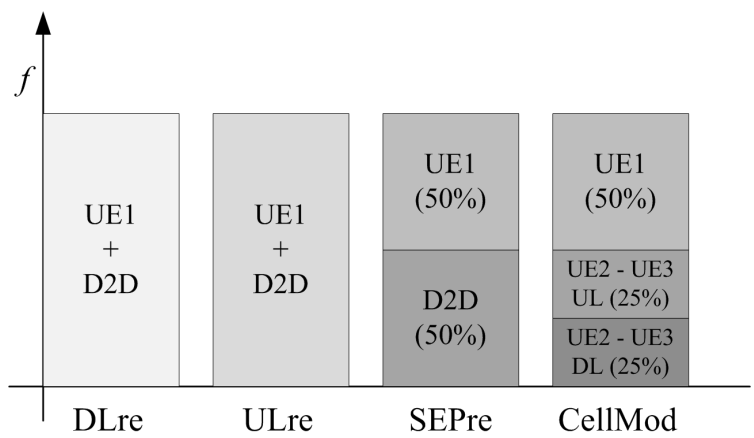

Figure 4. Illustration of resource allocation of considered resource sharing methods. In DLre and ULre modes, the cellular user and the D2D users operate in the same resource. In SEPre and CellMod modes, the cellular user and the D2D users occupy different resources.

Figure 1, and discuss the best resource allocation scheme out of the four possible modes. We assume one cellular user (UE1) and two D2D users (UE2 and UE3) sharing the available radio resources. For simplicity, we consider only distance-dependent pathloss, but no fading. Specifically, we consider the single-slope pathloss model [11] with pathloss exponent 4 :

$$
P(d)=\frac{P\left(d_{0}\right)}{d^{4}}
$$

where $P(d)$ denotes the received power at the distance $d$ from the transmitter and $P\left(d_{0}\right)$ is the received power at reference distance $d_{0}$. To adapt the normalized cell considered in our environment, we simply replace $P\left(d_{0}\right)$ with the transmit power. This channel model enables a one-to-one mapping between the distance of a channel link and the received signal strength. In addition, since the considered channel model provides the mean channel condition in a fading channel, the trend presented in this simplified model is consistent with the case where a more complex model is applied. We assume the distance between the D2D users and the BS to be $D$ and the distance between the two D2D users to be $L$. Assuming no power control, the transmit power is fixed to unity for the cellular transmission in UL and DL, and for the D2D user transmissions. Note that the per-RB transmit powers used in Section 4 are according to this scheme-the BS and the UE have the same (maximum) power spectral density. Under this channel model and the geometric constraint of the D2D users, the resource allocation decision depends on the cellular user (UE1) position only, under a given set of $D$ and $L$.

The interference caused by D2D users may come from any D2D users depending on which one is transmitting at the moment. Here, we assume the worst interference condition where the interference from D2D communication is caused by the user creating stronger interference.
The AWGN noise power is assumed to be the same as the signal power received at the cell border (i.e. $\mathrm{SNR}=0 \mathrm{~dB}$ at the cell edge). The metric for determining the resource sharing mode is the sum rate of the connection between UE2 and UE3, and of the cellular connection between BS and UE1. The sum rate takes into account either DL or UL resources depending on which one is shared with the D2D users. The sum rate is calculated by the Shannon capacity formula [12] according to the following equations

$$
\begin{aligned}
R_{\mathrm{ULre}}= & \log _{2}\left(1+\frac{P_{23}}{\max \left(P_{12}, P_{13}\right)+N_{0}}\right) \\
& +\log _{2}\left(1+\frac{P_{1}}{\max \left(P_{2}, P_{3}\right)+N_{0}}\right) \\
R_{\text {DLre }}= & \log _{2}\left(1+\frac{P_{23}}{\max \left(P_{2}, P_{3}\right)+N_{0}}\right) \\
+ & \log _{2}\left(1+\frac{P_{1}}{\max \left(P_{12}, P_{13}\right)+N_{0}}\right) \\
R_{\mathrm{SEPre}}= & \frac{1}{2} \log _{2}\left(1+\frac{P_{23}}{N_{0}}\right)+\frac{1}{2} \log _{2}\left(1+\frac{P_{1}}{N_{0}}\right) \\
R_{\text {CellMod }}= & \frac{1}{2} \cdot \frac{1}{4}\left(\log _{2}\left(1+\frac{P_{2}}{N_{0}}\right)+\log _{2}\left(1+\frac{P_{3}}{N_{0}}\right)\right) \\
& +\frac{1}{2} \log _{2}\left(1+\frac{P_{1}}{N_{0}}\right),
\end{aligned}
$$

where $P_{i}$ denotes the received power of the link between BS and UE $i$, and $P_{i j}$ denotes the received power of the link between UE $i$ and UE $j . N_{0}$ is the noise power at the receiver. The received power in each link is calculated by Equation (4).

The resource allocation scheme which gives the best sum rate is selected for each UE1 position according to

$$
R_{\max }=\max \left(R_{\mathrm{ULre}}, R_{\mathrm{DLre}}, R_{\mathrm{SEPre}}, R_{\text {CellMod }}\right)
$$

It should be noted that, without any further constraint, it may happen that either the cellular or the D2D connection is compromised in order to maximize the sum rate. For example, under the condition that UE1 is very close to BS, it is likely that the connection between the BS and the UE1 dominates the selection of the resource allocation scheme. The selected scheme might give the D2D connection little transmission rate. Similarly, when the D2D users are very close to each other and dominate the sum rate, the transmission rate of UE1 may be very limited.

Figure 5 shows the share of cell area where one specific resource allocation scheme is selected as the best one, under different values of $D$ and $L$. The curves 


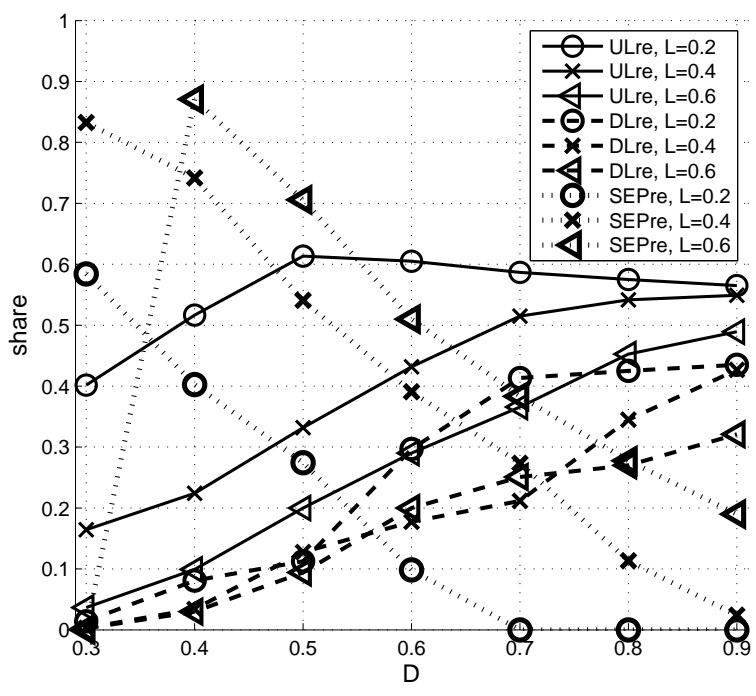

Figure 5. Share of cell area where one specific resource allocation scheme is selected as the best one, for different values of $D$ (the distance between BS and the D2D pair) and $L$ (the distance between the two D2D users). D2D communication is always beneficial for the system except for the case when the D2D users are at opposite sides with respect to $B S$ (i.e. $D=0.3$ and $L=0.6$ ), where none of ULre, DLre and SEPre resource allocation schemes occupies significant percentage area.

corresponding to CellMod mode are missing because it is selected only under the condition that the two D2D users are at opposite sides with respect to $\mathrm{BS}(L \approx 2 D)$. In this special condition, the CellMod mode is the favorable resource sharing scheme. This can be observed from Figure 5 by noticing that the share of all curves with $L=0.6$ goes to approximately 0 at $D=0.3$. However, except for this particular case, D2D communication is always beneficial for the system.

When the D2D users are further away from BS (i.e. when $D$ is large), the percentage area where the cellular user experiences strong interference reduces. It suggests the benefit of using non-separate resource sharing schemes (ULre or DLre) which provide higher resource usage efficiency. When $D$ is small, it is more beneficial to use either ULre or SEPre depending on the value of $L$. In small $D$ and small $L$ scenario, the signal strength between the D2D users is very strong. The ULre mode outperforms DLre mode in this case because the interference observed by the D2D users is smaller in ULre mode, which significantly improves channel quality of D2D communication.

Figure 6 shows the rate ratio of D2D communication to the CellMod mode under the parameter $D=0.7$ and $L=0.2$. The two circular spots give the position of two D2D users. The cellular user is at one given position in the cell, and the color at that position represents the rate gain from $\mathrm{D} 2 \mathrm{D}$ communication, which is the rate ratio

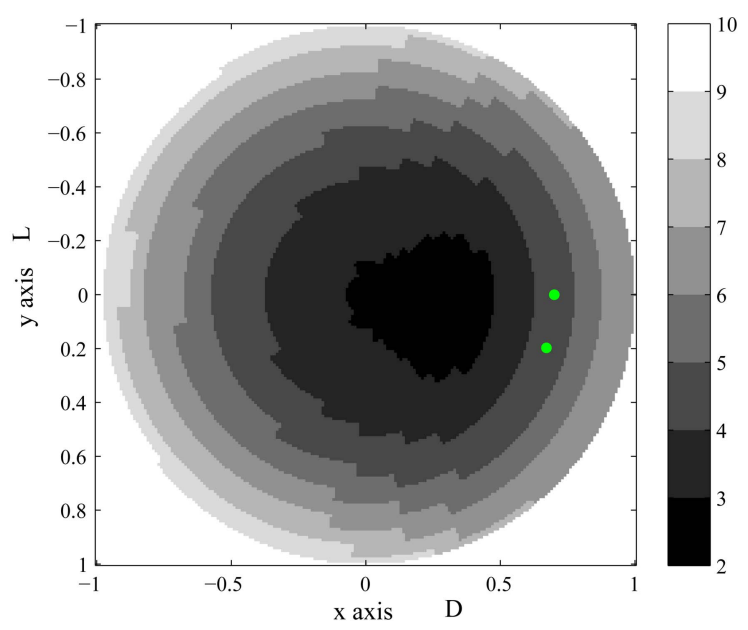

Figure 6. Rate ratio to CellMod mode with $D=0.7$ and $L=0.2$. The two circular spots denote the position of D2D users. The cellular user is at a given position in the cell and the background color at the position displays the rate ratio of the best resource sharing scheme to the CellMod mode.

between the rates obtained from the best resource sharing scheme and the CellMod mode. The area outside the unit circle is out of the considered cell and should not be considered. The gain is significant and depends on the position of the cellular user. In Figure 7, we illustrate the rate gain averaged over the considered single-cell with respect to different geometry of D2D users. It is clear that, in average, the larger $D$ and the smaller $L$ are beneficial for the system performance. Consistent with Figure 5, the rate gain vanishes when the D2D users are at opposite sides of BS (e.g. $D=0.3$ and $L=0.6$ ).

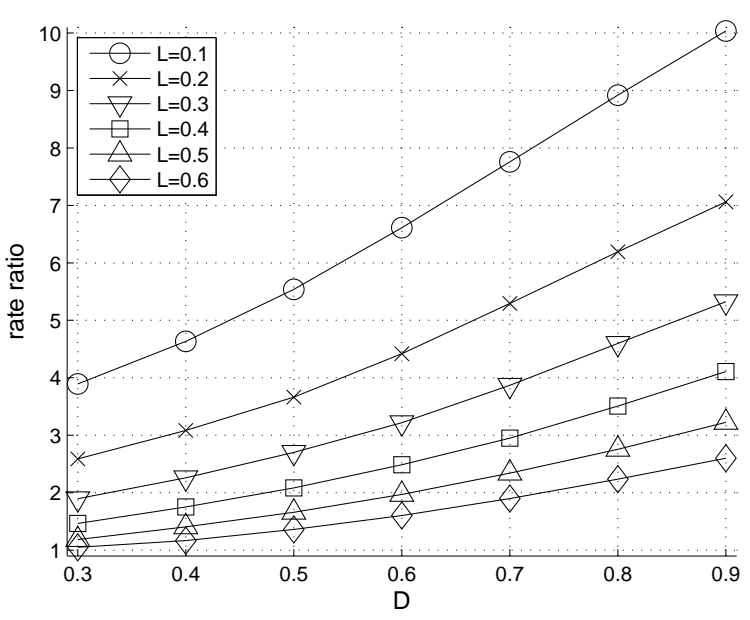

Figure 7. Average rate ratio over CellMod. The rate ratio is obtained by comparing the rate obtained from the best resource sharing scheme and the rate obtained from the CellMod mode. For different values of $D$ and $L$, the rate gain is averaged over the whole cell. 


\section{Indoor D2D as Underlay to a Metropolitan Area Network}

In the previous sections we have demonstrated that D2D communication can take place in an interference-limited network as well as the potential gains from D2D communication in a single cell. Now we consider D2D as an underlay to a metropolitan area network.

The cellular BS are deployed outdoors and outdoor cellular users share downlink resources with indoor D2D connections. The BSs are deployed in a multi-cell environment and the results are obtained from the center cell. The BS deployment is modelled by the well known Manhattan grid and follows the UMTS 30.03 recommendation [13] and the corresponding channel and path-loss models can be found in [9]. The D2D pairs are randomly generated within the same building block and the pathloss between them is below $90 \mathrm{~dB}$ which corresponds

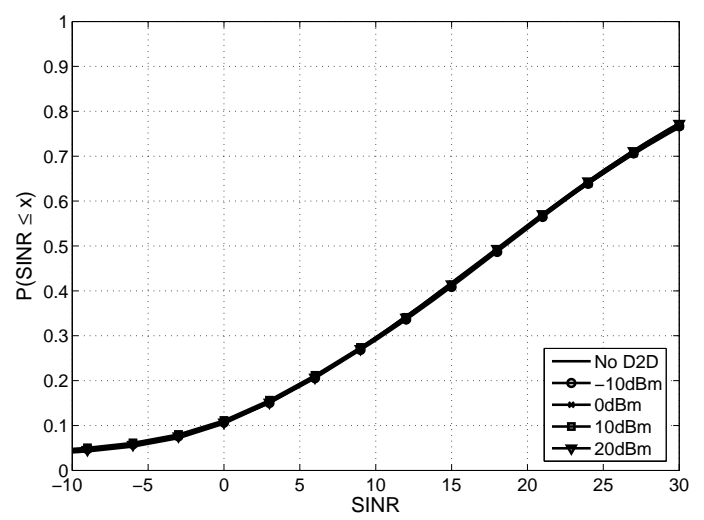

(a) Empirical CDF of cellular SINR for different D2D transmission power. There is no visible impact from the D2D communication on the SINR of the cellular network.

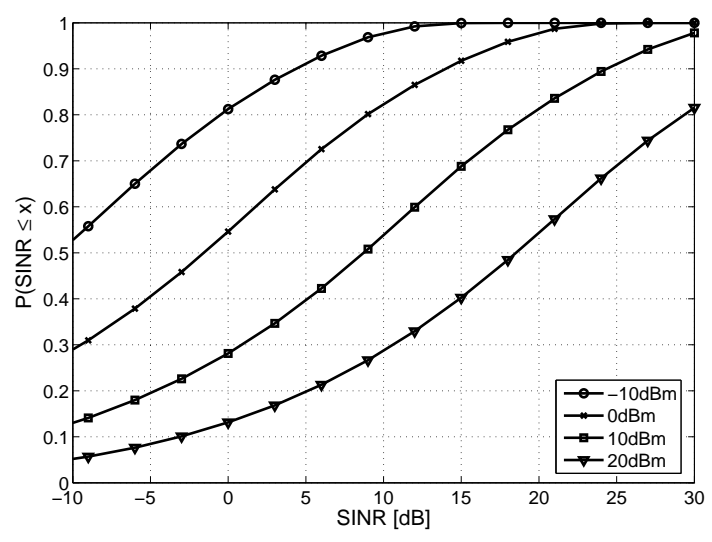

(b) Empirical CDF of D2D connection SINR for different D2D transmission power.

Figure 8. SINR of cellular and D2D connections sharing the cellular downlink resources. to a distance of up to $25 \mathrm{~m}$. The penetration loss of at least $14 \mathrm{~dB}$ through the outside wall of the building and the favorable propagation between outdoor BS and cellular devices in the same street isolates the indoor D2D connections from the outer cellular network.

Both the cellular devices and the D2D devices operate with full buffers, i.e. both the cellular network and the D2D devices utilize the full bandwidth with $100 \%$ load. A single OFDMA resource block (RB) is shared by one cellular user and one D2D pair in the cell.

The transmit power of the cellular BS is set to $37 \mathrm{dBm}$ and the transmit power of the D2D devices was limited to $20 \mathrm{dBm}$. Figure 8 illustrates the potential for D2D connection with different transmit power in such a scenario. The cellular SINR is not affected by the indoor D2D connections even when they transmit with $20 \mathrm{dBm}$. About $90 \%$ of the D2D connections experience a higher SINR than 0dB which we see as a lower threshold where D2D communication makes sense. In general the BS will also serve indoor users and the example might be overly optimistic. Nevertheless the BS can for example allocate only part of the downlink resources to D2D connections and schedule only outdoor cellular users in these resources. The BS can for example classify outdoor users based on path-loss, spatial signature or location information.

\section{Conclusions}

In this paper we analyze Device-to-Device (D2D) communications underlaying a cellular network. We show that given proper power control and coordination mechanisms it is possible to have D2D connections that reuse cellular band and still cause only minimal interference to the cellular network.

We propose a power control scheme for the D2D links that share uplink resources with a cellular network. In this case the maximum power that can be used for the D2D link is defined by taking the cellular uplink power control information as reference. We evaluate the proposed power control scheme in system simulations. The results show that by properly defining the maximum power on the D2D link a good D2D link SINR is achieved while at the same time the impact on the cellular network is minor. Thereby D2D communication can take place in interference-limited networks with full load, where a cognitive radio would not be able to detect a white space.

Further, we performed semi-analytical studies on a single-cell scenario to analyze how much gain can be expected from D2D communications. We considered several allocation strategies, including traditional cellular communications. The results show that significant gains in sum rate can be achieved by enabling D2D communications compared to the conventional cellular system. 
Finally, we showed in system simulations that indoor D2D communication causes negligible interference to outdoor cellular users in the downlink of a metropolitan area network.

\section{References}

[1] S. Haykin, "Cognitive radio: Brain-empowered wireless communications," IEEE Journal on Selected Areas in Communications, Vol. 23, No. 2, pp. 201-220, February 2005.

[2] J. Mitola and G. Q. Maguire Jr., "Cognitive radio: Making software radios more personal," IEEE Personal Communications, Vol. 6, No. 4, pp. 13-18, August 1999.

[3] I. F. Akyldiz, W. -Y. Lee, M. C. Vuran, and S. Mohanty, "Next generation dynamic spectrum access cognitive radio wireless networks: A survey," Computer Networks: The International Journal of Computer and Telecommunications Networking, Vol. 50, No. 13, pp. 2127-2159, September 2006.

[4] C. -K. Toh, M. Delwar, and D. Allen, "Evaluating the communication performance of an ad hoc wireless network," IEEE Transactions on Wireless Computing, Vol. 1, No. 3, pp. 402-414, July 2002.

[5] Y. Xue, B. Li, and K. Nahrstedt, "Optimal resource allocation in wireless ad hoc networks: A price-based ap- proach," IEEE Transactions on Mobile Computing, Vol. 5, No. 4, pp. 347-364, April 2006.

[6] ETSI, "BRAN; HIPERLAN2 type 2; data link control (DLC) layer; part 4: Extension for home environment," TS 101 761-4, v1.3.2, 2002.

[7] ETSI, "Terrestrial trunked radio (TETRA); voice plus data (V+D) designers' guide; part 3: Direct mode operation (DMO)," TR 102 300-3 v1.2.1, 2002.

[8] H.-Y. Hsieh and R. Sivakumar, "On using peer-to-peer communication in cellular wireless data networks," IEEE Transactions on Mobile Computing, Vol. 3. No. 1, pp. 57-72, January-February 2004.

[9] WINNER II D1.1.2, "WINNER II channel models," https:// www.ist-winner.org/deliverables.html, September 2007.

[10] 3GPP TR 25.814 V7.1.0, "Technical specification group radio access network; physical layer aspects for evolved universal terrestrial radio access (UTRA)," http://www. 3gpp.org/, September 2006.

[11] T. S. Rappaport, "Wireless communication principles and practice,” New Jersey: Prentice Hall, 1996.

[12] T. M. Cover and J. A. Thomas, "Elements of information theory," New York: Wiley, 1991.

[13] ETSI, "Recommendation TR 30.03 selection procedure for the choice of radio transmission technologies of the UMTS," 1997. 